\title{
Nail lichen planus: a patient with atypical presentation
}

\author{
Ahu Yorulmaz, Pelin Demirel Bulut, Basak Yalcin \\ Ankara Numune Research and Education Hospital, Department of Dermatology, Ankara, Turkey \\ Corresponding author: Dr. Yorulmaz Ahu, E-mail: ahuyor@gmail.com
}

\begin{abstract}
Lichen planus is a common chronic inflammatory dermatosis characterized by small, violaceous, flat-topped polygonal papules mostly seen on the flexural wrists and ankles. Several variants of lichen planus have been described based on either morphological appearance, configuration of the lesions or site of the involvement. Moreover, lichen planus is a distinctive dermatosis, in which mucosal or nail involvement may develop in the absence of cutaneous involvement. It has been estimated that nails are affected up to $10 \%$ of all patients with lichen planus. The most specific and unique nail finding of lichen planus is the pterygium formation. Pterygium unguis also known as dorsal pterygium refers to a V-shaped extension of the proximal nail fold over the nail plate, which eventually produces permanent onychatrophy. Here, we describe a patient with pterygium unguis, who also manifests atypical mucosal and cutaneous lesions of lichen planus.
\end{abstract}

Key words: Lichen planus; Nail; Pterygium; Onychatrophy; Oral

\section{INTRODUCTION}

Lichen planus is a common disease affecting up to $1 \%$ of the population. Lichen planus is a chronic inflammatory dermatosis with characteristic clinical and histopathological features. Classic lichen planus typically characterized by small, violaceous, flattopped polygonal papules mostly seen on the flexural wrists and ankles. A unique feature of lichen planus is superimposed, lacy, reticular pattern of crisscrossed whitish lines, termed "Wickham's striae", of which visualization is accentuated by application of a drop of immersion oil. Other than classic lichen planus, several variants of lichen planus, which differ in morphology and localization have been described. Moreover, lichen planus is a particular disease, in which mucosal or nail involvement may develop in the absence of cutaneous lesions. Although several nail changes are observed in lichen planus, one of the most distinguishing nail finding is the formation of dorsal pterygium [1-3]. Here, we report a case with dorsal pterygium, who also manifests atypical mucosal and cutaneous lesions.

\section{CASE REPORT}

A 62-year-old man came to our outpatient clinic with a several-month history of purplish red lesions over his trunk. While his family history was unremarkable, past medical history revealed diagnoses of bronchiectasis and atherosclerosis. He had gone through bypass surgery seven years earlier. The main complaint of the patient was two purplish red lesions, one of which was on the proximal end and the other was on the distal end of the thoracotomy incision scar. The patient also had dystrophic nails for twenty years. Medication history revealed he had been receiving metoprolol $50 \mathrm{mg}$, aspirin $100 \mathrm{mg}$ and clopidogrel $75 \mathrm{mg}$ daily for the previous seven years. Upon dermatological examination, we observed two violaceous patches located both ends of the thoracotomy incision scar. The one on the proximal end had a darker peripheral rim and the one on the distal end had a scale, which was centrally adherent (Fig. 1). Dermatological examination also revealed adhesion between the epidermis of the dorsal nail fold and the nail bed on the right third, fourth and fifth, also left first, third and fourth fingers. Complete

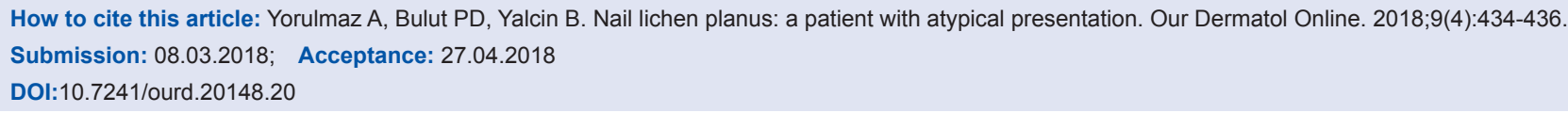


anonychia of the left fourth fingernail was also detected (Figs 2-4). Oral mucosal examination demonstrated a slightly elevated whitish annular lesion (Fig. 5). Based on the clinical findings we made a diagnosis of lichen planus with pterygium formation. We suggested the patient to perform skin and oral mucosal lesion biopsy. However, the patient did not want any invasive procedure. Laboratory studies including complete blood count and differential, erythrocyte sedimentation rate and a complete serum chemistry profile revealed no abnormalities. Serologic tests for hepatitis B, C, and human immunodeficiency virus were negative with positive results for anti-HBs antibody. We prescribed topical corticosteroids for skin lesions and suggested intralesional injections of corticosteroids for nail involvement. On the other hand, the patient only accepted topical treatment and lost follow-up.

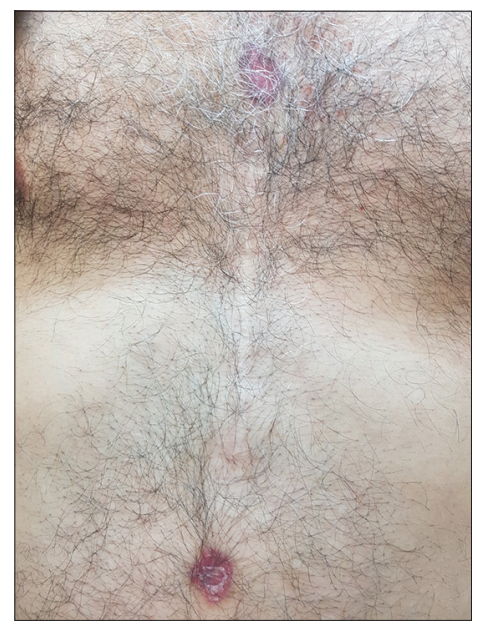

Figure 1: Violaceous patches located both ends of the thoracotomy incision scar. The one on the proximal end had a darker peripheral border and the one on the distal end had a scale, which was centrally adherent.

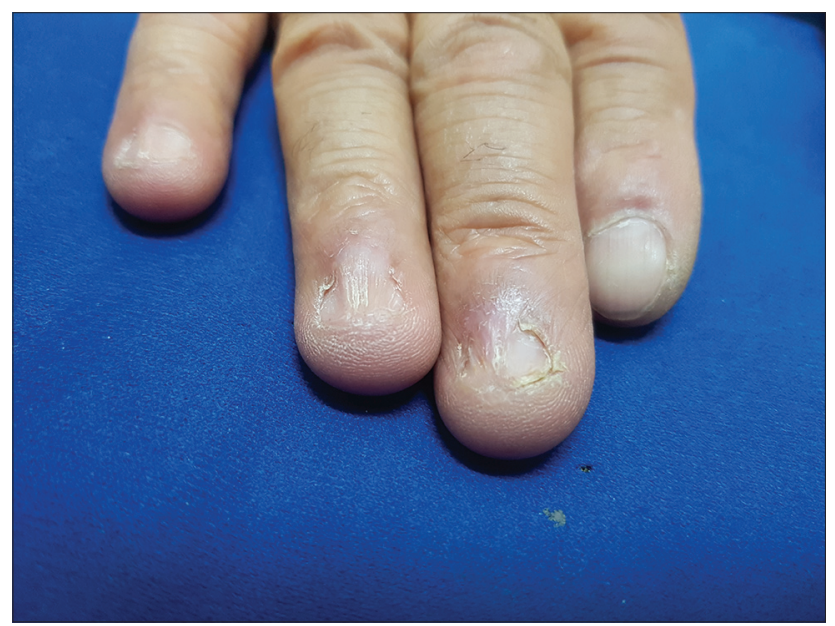

Figure 2: V-shaped extension of the proximal nail fold epidermis over the nail plate causing partial destruction of the right third, fourth and fifth fingernails.

\section{DISCUSSION}

It has been estimated that nails are affected up to $10 \%$ of all patients with lichen planus. Lichen planus has been associated with several nail findings, including lateral

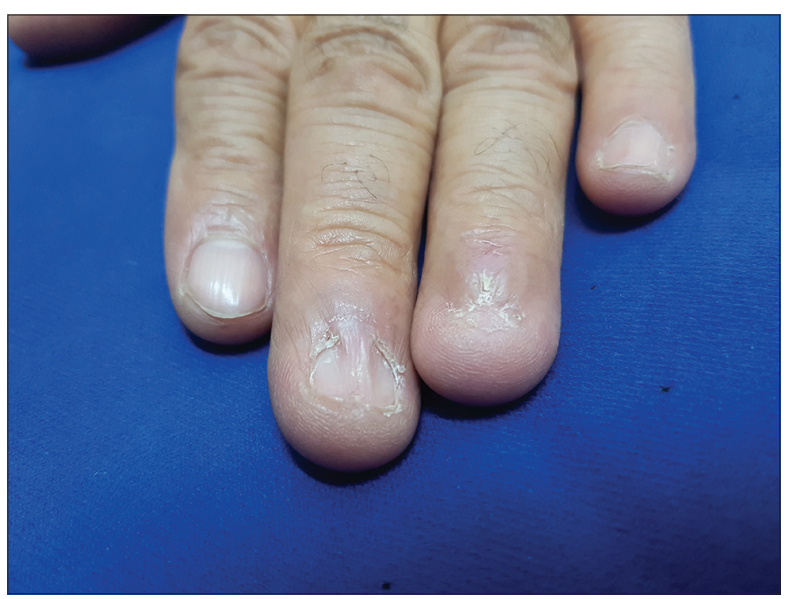

Figure 3: Adhesion between the epidermis of the dorsal nail fold and the nail bed on the left third digit and onychatrophy of the left fourth fingernail.

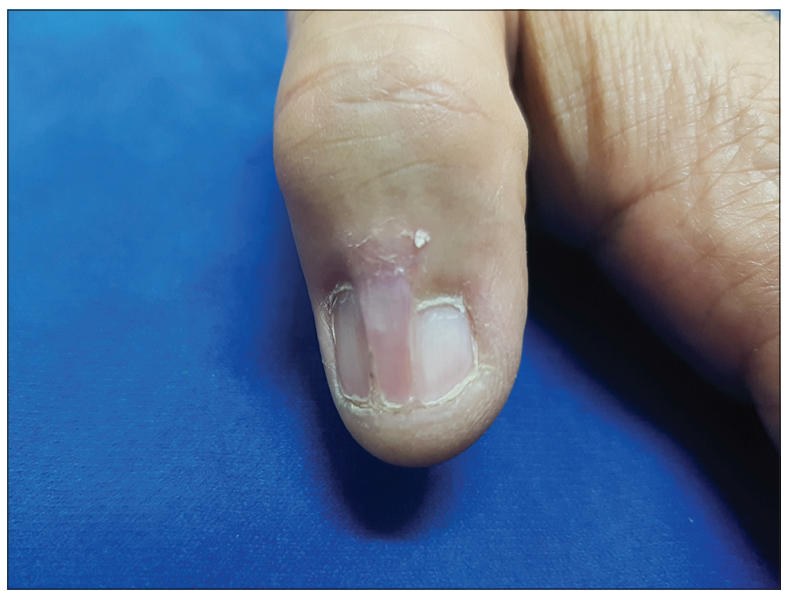

Figure 4: Extension of the proximal nail fold over the nail plate creating split portions on the nail plate on the left thumb.

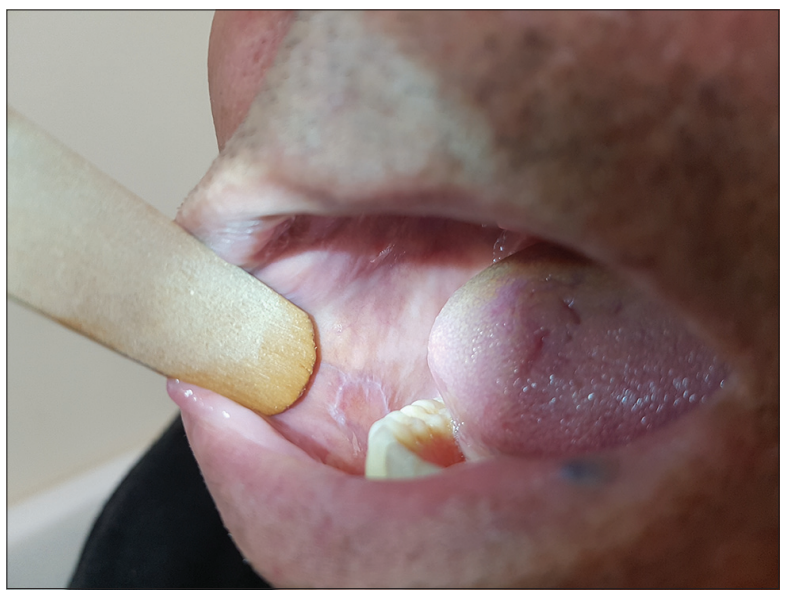

Figure 5: An annular lesion with a slightly elevated whitish rim located on the right buccal mucosa. 
thinning, longitudinal ridging and striations, fissuring, distal splitting, subungual hyperkeratosis, onycholysis, trachyonychia and erythematous patches of the lunula. Pterygium unguis is the most specific and unique nail finding of lichen planus. It has been defined as wingshaped scar formation, which is a result of severe nail matrix damage. Lichen planus is a chronic inflammatory disease. If the entire lenght of the nail matrix is involved in the inflammatory process, permanent destruction of the nail matrix eventually gives rise to pterygium formation. This specific sign is typically characterized by a $\mathrm{V}$-shaped extension of the proximal nail fold fusing with the nail bed. Subsequently gradual destruction of the nail causes onychatrophy $[2,4-8]$.

In lichen planus, fingernails are affected more commonly than toenails. The number of involved nails differs in every single patient. Although it is known that in most of the patients nail lesions develop simultaneously in all involved digits, it is quite interesting that the severity of the disease may vary in degree from nail to nail and within the same nail. In some patients, though initially only few digits are affected, subsequent involvement of other digits is frequently observed. Most of the nail findings of lichen planus are not exclusively seen in lichen planus. Ridging and splitting, for instance, quite common in the elderly without any dermatological disease [5]. However, pterygium is the most specific finding for nail lichen planus. Although it can be seen in other conditions, including trauma, Raynaud phenomenon, peripheral vascular disease, radiotherapy and immunobullous diseases, pterygium has been accepted as the almost pathognomonic clinical sign for nail lichen planus [8]. When differentiating nail lichen planus from other diseases causing pterygium, simultaneous involvement of several digits is the key point for the diagnosis, since in other diseases contemporaneous involvement is unexpected $[5,8]$.

Our patient represents typical pterygium formation. Although nail biopsy remains the gold standard for the diagnosis of nail lichen planus, simultaneous involvement of several digits with $\mathrm{V}$-shaped extension of the proximal nail fold over the nail bed and anonychia spontaneously directed us to the diagnosis of pterygium. We have presented our patient not only because pterygium is the most specific and almost pathognomonic nail finding of lichen planus, but also mucosal and cutaneous features of our patient were atypical. Configuration of the mucosal lesion, which was annular with slightly elevated whitish interlacing keratotic lines and localization of the violaceous patches, which were both ends of the thoracotomy incision scar were exceptionally interesting. The patient told that nail lesions had begun twenty years earlier, cutaneous lesions began several months ago and he did not know when the oral mucosal lesion had begun. It is known that pterygium does not necessarily correlate with the duration of the disease [5]. Thus, we think that in our patient lichen planus had begun twenty years ago but tended to be chronic, with fluctuations in signs and symptoms over time. Moreover, in our opinion, medications of the patient, which were metoprolol, acetylsalicylic acid and clopidogrel might have played a role in aggravating the condition, since these drugs are potential triggers for lichen planus $[1,9,10]$. On the other hand, the main limitation of our case report is that, we could not prove the diagnosis of lichen planus with histopathology. We suggest further case reports to be presented to enlighten atypical presentations of lichen planus with histopathological findings.

\section{CONSENT}

The examination of the patient was conducted according to the Declaration of Helsinki principles.

\section{REFERENCES}

1. Weston G, Payette M. Update on lichen planus and its clinical variants. Int J Womens Dermatol. 2015;1:140-9.

2. Lehman JS, Tollefson MM, Gibson LE. Lichen planus. Int J Dermatol. 2009;48:682-94.

3. Wagner G, Rose C, Sachse MM. Clinical variants of lichen planus. J Dtsch Dermatol Ges. 2013;11:309-19.

4. Piraccini BM, Saccani E, Starace M, Balestri R, Tosti A. Nail lichen planus: response to treatment and long term follow-up. Eur J Dermatol. 2010;20:489-96.

5. Tosti A, Peluso AM, Fanti PA, Piraccini BM. Nail lichen planus: clinical and pathologic study of twenty-four patients. J Am Acad Dermatol. 1993;28:724-30.

6. Baran LR. Yellow nail syndrome and nail lichen planus may be induced by a common culprit. focus on dental restorative substances. Front Med (Lausanne) 2014;1:46.

7. Goettmann S, Zaraa I, Moulonguet I. Nail lichen planus: epidemiological, clinical, pathological, therapeutic and prognosis study of 67cases. J Eur Acad Dermatol Venereol. 2012;26:1304-9.

8. Murynka T, Prajapati V, Barankin B. Dermacase. Can you identify this condition? Nail lichen planus. Can Fam Physician. 2009;55:1207-8.

9. Guijarro Guijarro B, López Sánchez AF. Lichenoid reaction caused by Clopidogrel, a new anti-platelet drug. Med Oral. 2003;8:33-7.

10. Ruiz Villaverde R, Blasco Melguizo J, Mendoza Guil F, Martín Sánchez MC, Naranjo Sintes R. Generalized lichen planus-like eruption due to acetylsalicylic acid. J Eur Acad Dermatol Venereol. $2003 ; 17: 470-2$.

Copyright by Yorulmaz Ahu, et al. This is an open-access article distributed under the terms of the Creative Commons Attribution License, which permits unrestricted use, distribution, and reproduction in any medium, provided the original author and source are credited.

Source of Support: Nil, Conflict of Interest: None declared. 